\title{
Biometric Identification using Human Ear
}

\author{
Aishna Sharma, Natasha Lalwani, Mani Roja M. Edinburgh
}

\begin{abstract}
Biometrics refers to the metrics of the human characteristics which has gained much popularity in recent times. It is a form of identification and access control. Widely used forms of biometrics are facial recognition, finger print recognition, iris recognition, etc. but the drawback is that most of these features change over time. The human ear is a cogent source of data to classify biometrically since its attributes do not change substantially as time progresses. This paper explores the field of ear biometric wherein the database images are re-sized to $128 \mathrm{x}$ 256 pixels and then converted to grayscale image. Various transforms viz. Discrete Cosine Transform, Discrete Fourier Transform, Discrete Wavelet Transform are then applied to extract the features. The coefficients of the test image are compared with the coefficients of the registered database image. On comparison, Euclidean distance classifier is used to recognize the test image from the database. The database used consists of 25 subjects with 6 images per person out of which the initial 4 images are used to train the model, and the remaining 2 for testing. The outputs of various transforms were compared and the best accuracy obtained is $86 \%$ using Discrete Wavelet Transform.

Keywords: Discrete Cosine Transform, Discrete Fourier Transform, Discrete Wavelet Transform, Ear Recognition, Person Identification
\end{abstract}

\section{INTRODUCTION}

Personal identification is more relevant than it was before in different sectors and hence biometrics has been receiving a lot of attention lately as it deals with the identification of individuals based on various characteristics. It is conventionally perceived that biometric has become a essential element of the identification technology. Biometrics can be bifurcated into two categories, namely physiological and behavioural. The physiological class is based on external physical characteristic measurements, such as height, body shape, face shape, hand shape, ear shape, odour, retina, iris, deoxyribonucleic acid, fingerprint etc. The behavioural class measures the learned behaviours such as body posture, speech, handwriting, heartbeat, eye blinking pattern, etc. However, in due course of time the identifying factors tend to change and cause hindrances in the identification process. Hence, all the commercially used techniques have their shortcomings.

Revised Manuscript Received on October 30, 2019

* Correspondence Author

Natasha Lalwani*, Department of Electronics and Telecommunication Engineering, Thadomal Shahani Engineering College, Mumbai, India. Email: natashalalwani90@gmail.com

Aishna Sharma, Department of Electronics and Telecommunication Engineering, Thadomal Shahani Engineering College, Mumbai, India. Email: aishna06@gmail.com

Dr. Mani Roja M. Edinburgh, Department of Electronics and Telecommunication Engineering, Thadomal Shahani Engineering College, Mumbai, India. Email: maniroja@thadomal.org

(C) The Authors. Published by Blue Eyes Intelligence Engineering and Sciences Publication (BEIESP). This is an open access article under the CC BY-NC-ND license (http://creativecommons.org/licenses/by-nc-nd/4.0/)
Prior research reveals that human ear is one of the representative human biometrics with uniqueness and stability since the deformation of ears due to ageing and weight is very little over a long period of time. According to documented history, French Criminologist Alphonse Bertillon in 1890 was the first to use the ear for identification. The most widely cited methods used for ear biometrics was presented by Burge and Burger in 1997 [1]. Several feature extraction and matching schemes have been proposed by researchers since then. These vary from simple appearance-based methods such as principal component analysis (PCA) to more complex methods based on scale-invariant feature transforms (SIFT), local binary patterns, wavelet transforms and force fields.

Burge and Burger devised the first well known technique for ear detection. Using deformable contours, they positioned the ear on a Gaussian pyramid representing the gradient of the side profile. However, this technique requires user interaction for contour initialization. Ansari and Gupta (2007) [2] had a similar approach where they detected the ear from the edges of outer helices. They achieved an accuracy of about 93.34\% with this approach. Moreno et al (1999) [3] were one of the firsts to introduce an automated system for recognition of ears. They extracted features by a compression network, and included them in their feature vector along with outer ear points, ear shape, and wrinkles. Tariq et al (2011) [4] extracted the features of a ear using the Haar wavelet, and matched them using fast normalized cross correlation (NCC). They achieved a recognition rate as high as $98.33 \%$; however it goes to $95.2 \%$ with a database with more subjects. Feng and $\mathrm{Mu}$ (2009) [5] combined wavelet transform and local binary pattern operators, resulting in an accuracy of $96.86 \%$ using chi-squared distance. Wang et al (2008) [6] also used a similar approach; they decomposed ear images using Haar wavelet transform, followed by applying uniform local binary patterns. The resulting recognition rate was as high as $100 \%$, but deteriorated to around $42.41 \%$ with change in angles. Karuna et al (2015) [7] used Gabor filter (GF) for feature extraction, and further used discrete wavelet transform (DWT) to filter the Gabor feature matrix of its redundancies, achieving a recognition rate of $90 \%$. Abate et al (2006) [8] extracted features using rotation invariant descriptors viz. Generic Fourier Descriptors. They got an accuracy of $96 \%$. Taertutulakarn et al (2016) [9] used the geometric features on 3D ear surface for a 2D ear image, further used PCA for feature extraction and attained a recognition rate of $92 \%$ of 50 volunteers. Zhang et al (2013) [10] used Gabor filters for feature extraction. Their method uses the scale information as the feature, avoiding the discrepancies faced in most Gabor based feature extraction methods. They achieved a recognition rate of $90 \%$. Khobragade et al (2015) [11] used MATLAB as the image processing tool and worked on image enhancement, filtering and feature extraction. 


\section{Biometric Identification using Human Ear}

The ear recognition experiment by Yuan et al (2007) [12] made use of 2 sets of ear images, subset 1 and subset 2 , containing profile views of 79 subjects. The subject images from a subset were used to train, and to get the feature space with the FSLDA (Full-space Linear Discriminant Analysis). The recognition dropped as the rotation angle increased. Maximum accuracy of $90 \%$ was obtained. Tiwari et al (2015) [13] proposed a unique approach for the automatic ear recognition for the newborn using 2D imaging, GF and HAAR. They worked on a database of 210 subjects with 10 images per person out of which the initial 4 images were used for training and the remaining 6 for testing. An accuracy of 89.285 was obtained. The ear recognition experiment by Yan et al (2007) [14] uses an approach based on Iterative Closest Point (ICP) for 3D shape matching. The accuracy achieved is $97.6 \%$ and $94.2 \%$ for subjects with earrings. Yuizono et al (2002) [15] introduced a recognition system for 2D intensity images of the ear using genetic search. An approximate recognition rate of $100 \%$ was achieved, and a rejection rate for unknown samples was also reported to be $100 \%$. Hurley et al (2007) [16] used force field transformation to extract features. A classification rate of $99.2 \%$ was achieved on their dataset.

Alvarez et al (2012) [17] introduced a new method combining geodesic active contours and ovoid model, which were then used to compare subjects in a way independent of the location and size of the ear. Arunachalam and Alagarsamy (2017) [18] proposed an algorithm for ear recognition using band limited phase only correlation (BLPOC) based local block matching. Experimental analysis has been performed on a dataset of nearly 50 images. Oravec and Pavlovicova (2004) [19] created an application based on Local Binary Patterns (LBP) and Principal Component Analysis (PCA) algorithms for feature extraction. With the use of LBP extraction algorithm, maximum recognition rate of $100 \%$ was obtained, whereas with the use of PCA extraction algorithm, maximum recognition rate of $60 \%$ was obtained. Anwar et al (2015) [20] suggested an algorithm based on scale-invariant feature transform (SIFT) for ear recognition. The proposed algorithm was applied on two databases and the accuracies of $95.2 \%$ and $100 \%$ were achieved.

\section{PROPOSED METHOD}

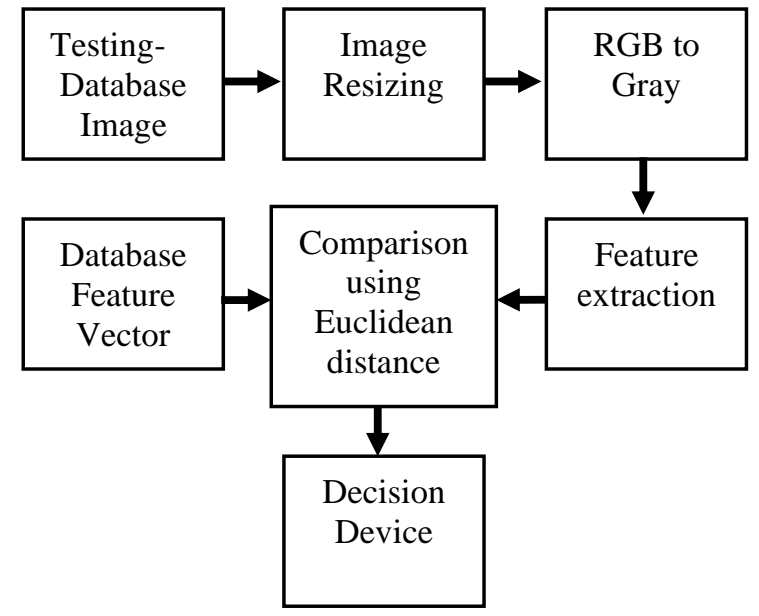

Fig. 1. Block diagram of ear identification system.

\section{A. Discrete Cosine Transform}

Discrete Cosine Transform (DCT) is an essential technique or method to convert a signal into elementary frequency component. It describes a finite sequence of data points with respect to the sum of cosine functions oscillating at various frequencies. Implementing cosine functions instead of sine functions is critical for compression, since a fewer cosine functions are needed to approximate a typical signal, whereas the cosines express a particular choice of boundary conditions for differential equations. Discrete Cosine Transform helps partition the image into parts (or spectral sub-bands) of significance (with respect to the image's visual quality). It is analogous to Discrete Fourier Transform (which involves both sine and cosine functions), it converts a signal or image from spatial domain to frequency domain.

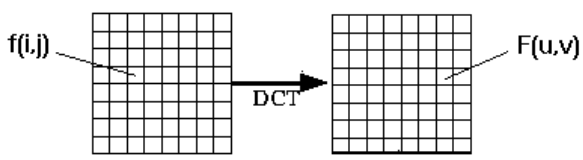

Fig. 2. Discrete Cosine Transform

In formal terms, discrete transformation of cosine is a linear, invertible function $\mathrm{f}: \mathbb{R}^{\mathrm{N}} \rightarrow \mathbb{R}^{\mathrm{N}}$ (where $\mathbb{R}$ is denotes the set of real numbers), or likewise an $\mathrm{N}$ by $\mathrm{N}$ square matrix. The $\mathrm{N}$ real numbers $\mathrm{x}_{0}, \ldots, \mathrm{X}_{\mathrm{N}-1}$ are transformed into the $\mathrm{N}$ real numbers $\mathrm{X}_{0}, \ldots, \mathrm{X}_{\mathrm{N}-1}$ according to one of the formulas :

- Two Dimensional DCT Equation (DCT-II)

$$
F[u, v]=\frac{1}{N^{2}} \sum_{m=0}^{N-1} \sum_{n=0}^{N-1} f[m n] \cos \left[\frac{(2 m+1) u \pi}{2 N}\right] \cos \left[\frac{(2 n+1) v \pi}{2 N}\right]
$$

Where

$\mathrm{u}, \mathrm{v}=$ discrete frequency variables $(0,1,2, \ldots, \mathrm{N}-1)$,

$\mathrm{f}[\mathrm{m} \mathrm{n}]=\mathrm{N}$ by $\mathrm{N}$ image pixels $(0,1,2, . ., \mathrm{N}-1)$,

$\mathrm{F}[\mathrm{u}, \mathrm{v}]=\mathrm{DCT}$ result

- Two Dimensional IDCT Equation

$f[m n]=\sum_{m=0}^{N-1} \sum_{n=0}^{N-1} c[u] c[v] F[u, v] \cos \left[\frac{(2 m+1) u \pi}{2 N}\right] \cos \left[\frac{(2 n+1) v \pi}{2 N}\right]$

Where
$\mathrm{m}, \mathrm{n}=$ image result pixel indices $(0,1,2, \ldots, \mathrm{N}-1)$
$\mathrm{f}[\mathrm{m} \mathrm{n}]=\mathrm{N}$ by $\mathrm{N}$ IDCT result
$\mathrm{F}[\mathrm{u}, \mathrm{v}]=\mathrm{N}$ by $\mathrm{N}$ DCT result

DCT, and DCT-II in particular, is frequently used in signal and image processing, especially for loss compression, as it has a strong compaction property. Most of the signal data appears to be concentrated in a few DCT components with low frequencies. 
DCTs are also closely related to Chebyshev polynomials, and fast DCT algorithms are in Chebyshev approximated of arbitrary functions by series of Chebyshev polynomials. Other applications of DCT are DVD/Video CD players, Cable TV, HDTV, Ultrasound/MRI systems, Digital Camera etc.

\section{B. Discrete Fourier Transform}

Discrete Fourier Transform (DFT) is a very fast processing tool used in digital signal processing, convolution, filtering, image analysis, and more. It is used to transform an intensity image into the domain of spatial frequency. Discrete Fourier Transform is widely used as it is mathematically easier to analyze effects of transmission medium, noise, etc on simple sine functions, then add to get effect on complex signal. In order to study the spatial variations, an image is decomposed into a set of orthogonal functions. It is generally implemented using Fast Fourier Transform (FFT) algorithms as FFT saves time due to lesser complex calculations.
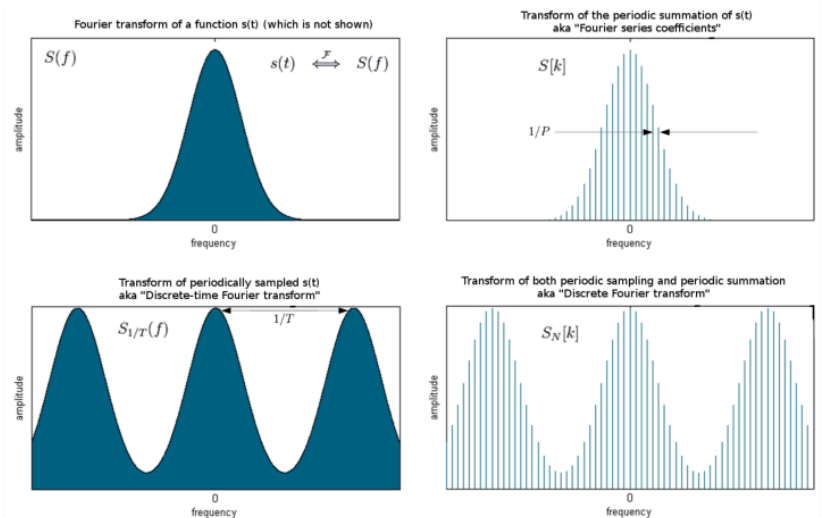

Fig. 3. Discrete Fourier Transform

The basic operation of Discrete Fourier Transform is similar to discrete cosine transform. The DCT matrix is replaced by the twiddle matrix with the help of 'twiddle factor' (W).

- Two Dimensional DFT Equation

$$
f[k, l]=\frac{1}{M N} \sum_{m=0}^{M-1} \sum_{n=0}^{N-1} f[m, n] e^{-j 2 \pi\left(\frac{k}{M} m+\frac{l}{N} n\right)}
$$

Where

$\mathrm{MxN}=$ size of $2 \mathrm{D}$ matrix of image pixels

$\mathrm{k}=0,1,2, \ldots, \mathrm{M}-1$

$\mathrm{l}=0,1,2, \ldots, \mathrm{N}-1$

$\mathrm{f}[\mathrm{m} \mathrm{n}]=\mathrm{N}$ by $\mathrm{N}$ image pixels $(0,1,2, \ldots, \mathrm{N}-1)$

$\mathrm{F}[\mathrm{k}, \mathrm{l}]=\mathrm{DFT}$ result

- Two Dimensional IDFT Equation

$$
f[m, n]=\sum_{k=0}^{M-1} \sum_{l=0}^{N-1} F[k, l] e^{j 2 \pi\left(\frac{k}{M} m+\frac{l}{N} n\right)}
$$

- Twiddle Factor (W)

$$
\mathrm{W}_{\mathrm{N}}=\mathrm{e}^{-\mathrm{j} 2 \pi / \mathrm{N}}
$$

Where $\mathrm{N}$ is the period of the signal

The Discrete Fourier Transform can calculate a signal's frequency spectrum, which is a direct examination of information encoded in the frequency, phase, and amplitude of the component sinusoids. The DFT can find a system's frequency response from the system's impulse response, and vice versa. This enables systems to be analysed in the frequency domain, just as convolution enables systems to be analysed in the time domain. The Discrete Fourier Transform can be used as an intermediate step in more elaborate signal processing techniques.

The DFT is commonly used in spectrograms, power spectral density estimation, matched filtering, and others. The DFT has lower energy compaction as compared to DCT, but it is preferred over DCT as it is faster, and involves lesser complex calculations.

\section{Discrete Wavelet Transform}

Discrete Wavelet Transform is a very adaptable signal processing tool where the signal is decomposed into wavelet coefficients and scaling function. Wavelets allow the analysis of both time and frequency simultaneously because the energy of wavelets is concentrated in time and still have characteristics similar to waves i.e. they are periodic. Wavelet representation yields a mathematical tool that helps in analysing transient, time-variant signals that are not predictable, statistically, especially at the area of discontinuities. Wavelets also convert the image into a series of wavelets whose storage is more efficient that that of pixel blocks. Discrete wavelet transform is an efficient and useful tool in which the wavelets are sampled discretely. Digital signals are decomposed into multiple sub-bands so that the sub-bands at lower frequencies have finer frequency resolution and coarser time resolution than the bands at higher frequencies.

\begin{tabular}{|c|c|}
\hline LL & HL \\
\hline LH & HH \\
\hline
\end{tabular}

\begin{tabular}{|c|c|c|}
\hline LL & HL & \multirow{2}{*}{ HL } \\
\cline { 1 - 1 } LH & HH & \\
\hline LH & HH \\
\hline
\end{tabular}

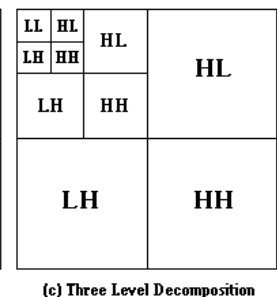

Fig. 4. DWT Decomposition levels

The Discrete Wavelet Transform has extensive applications in the fields of signal analysis. It is used in removing noise from electrical signals, in detecting anomalies, and in compressing data. Discrete Wavelet Transform is preferred because of its high flexibility i.e. it can be performed with multiple bases called wavelets. It is easier to filter in or filter out noise, as the signal is divided into many components.

Where

$\mathrm{m}, \mathrm{n}=$ image result pixel indices $(0,1,2, \ldots, \mathrm{N}-1)$

$\mathrm{f}[\mathrm{m}, \mathrm{n}]=\mathrm{M}$ by $\mathrm{N}$ IDFT result

$\mathrm{F}[\mathrm{k}, \mathrm{l}]=\mathrm{M}$ by $\mathrm{N}$ DFT result 


\section{IMPLEMENTATION}

The database used in this project comprises of 6 ear samples (3 pairs) each from 25 subjects, out of which 4 have been considered for the training database and 2 for testing the accuracy. The images used are of the size 128 x 256 pixels.

The resized images are converted to grayscale images, this is the pre-processing stage. The lineaments of the images are then obtained using any one of the transforms in the proposed methods i.e. DCT, DFT or DWT. With the help of zig-zag scanning (used to cluster low frequency coefficients in top of vector and high frequency coefficients at the bottom) we obtain a feature vector which is later compared with the feature vector of the testing database using Euclidean distance.

$$
\text { Euclidean Distance }=\sqrt{\sum_{i=0}^{n}\left(x_{i}-y_{i}\right)^{2}}
$$

\section{RESULT}
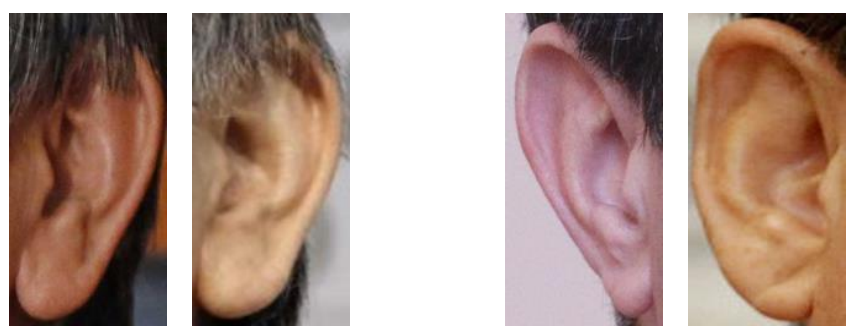

Fig. 5(a). Accepted Pair

Fig. 5(b). Rejected Pair

\section{A. Results using Discrete Cosine Transform}

Table- I: Results using Discrete Cosine Transform

\begin{tabular}{|c|c|c|}
\hline No. Of Coefficients & Accuracy (\%) & $\begin{array}{c}\text { Time Taken } \\
\text { (seconds) }\end{array}$ \\
\hline 8 & 67 & 1.87 \\
\hline 16 & 67 & 1.927 \\
\hline 32 & 70 & 1.903 \\
\hline 64 & 75 & 1.906 \\
\hline 128 & 75 & 1.856 \\
\hline 256 & 77 & 1.827 \\
\hline 512 & 80 & 2.982 \\
\hline 1024 & 83 & 2.951 \\
\hline
\end{tabular}

B. Results using Discrete Fourier Transform

Table- II: Results using Discrete Fourier Transform

\begin{tabular}{|c|c|c|}
\hline No. Of Coefficients & Accuracy (\%) & $\begin{array}{c}\text { Time Taken } \\
\text { (seconds) }\end{array}$ \\
\hline 8 & 65 & 1.734 \\
\hline 16 & 69 & 1.791 \\
\hline 32 & 70 & 1.746 \\
\hline 64 & 75 & 1.738 \\
\hline 128 & 78 & 1.800 \\
\hline 256 & 81 & 1.937 \\
\hline 512 & 81 & 1.938 \\
\hline 1024 & 80 & 2.232 \\
\hline
\end{tabular}

C. Results using Discrete Wavelet Transform (Level I)

Table- III: Results using Discrete Wavelet Transform (Level I)

\begin{tabular}{|c|c|c|}
\hline Wavelets & Accuracy (\%) & $\begin{array}{c}\text { Time Taken } \\
\text { (seconds) }\end{array}$ \\
\hline
\end{tabular}

\begin{tabular}{|c|c|c|}
\hline Haar & $\mathbf{6 8}$ & $\mathbf{1 . 8 0 8}$ \\
\hline Db10 & 57 & 1.199 \\
\hline Db40 & 79 & 3.317 \\
\hline Bior1.1 & 70 & 2.097 \\
\hline Bior3.9 & 52 & 2.446 \\
\hline Coif2 & 77 & 2.341 \\
\hline Coif4 & 67 & 2.140 \\
\hline
\end{tabular}

D. Results using Discrete Wavelet Transform (Level II)

Table- IV: Results using Discrete Wavelet Transform (Level II)

\begin{tabular}{|c|c|c|}
\hline Wavelets & Accuracy (\%) & $\begin{array}{c}\text { Time Taken } \\
\text { (seconds) }\end{array}$ \\
\hline Haar & 80 & 1.899 \\
\hline Db10 & 86 & 2.204 \\
\hline Db40 & 85 & 3.531 \\
\hline Bior1.1 & 83 & 1.439 \\
\hline Bior3.9 & 62 & 1.814 \\
\hline Coif4 & 76 & 2.502 \\
\hline Dmey & 86 & 2.072 \\
\hline Db35+Haar & 86 & 3.584 \\
\hline Db35+RBio1.5 & 83 & 3.625 \\
\hline
\end{tabular}

E. Results using Discrete Wavelet Transform (Level III) Table- V: Results using Discrete Wavelet Transform (Level III)

\begin{tabular}{|c|c|c|}
\hline Wavelets & Accuracy (\%) & $\begin{array}{c}\text { Time Taken } \\
\text { (seconds) }\end{array}$ \\
\hline Haar & 77 & 2.972 \\
\hline Db10 & 77 & 2.973 \\
\hline Db35 & 85 & 4.060 \\
\hline Db40 & 72 & 4.160 \\
\hline Bior1.1 & 78 & 1.234 \\
\hline Bior3.9 & 44 & 1.320 \\
\hline Coif2 & 78 & 3.096 \\
\hline Coif4 & 74 & 3.276 \\
\hline Dmey & 80 & 4.073 \\
\hline Haar+Db10+Bior1.1 & 75 & 1.898 \\
\hline Coif5+Bior6.8+Db35 & 75 & 3.130 \\
\hline
\end{tabular}

F. Comparison of Discrete Cosine Transform and Discrete Fourier Transform

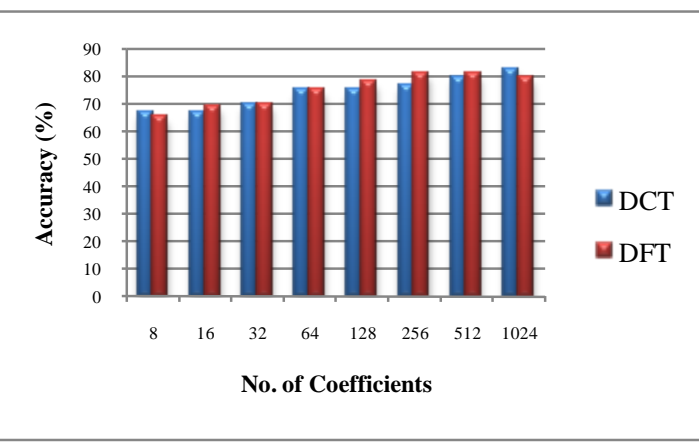

Fig. 6. Comparison of Discrete Cosine Transform and Discrete Fourier Transform

G. Comparison of Discrete Wavelet Transform Levels

Published By:

Blue Eyes Intelligence Engineering

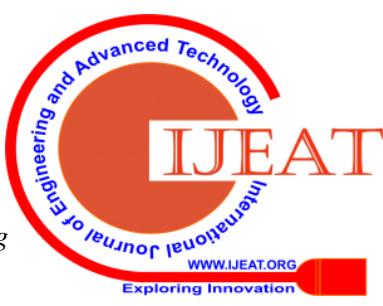




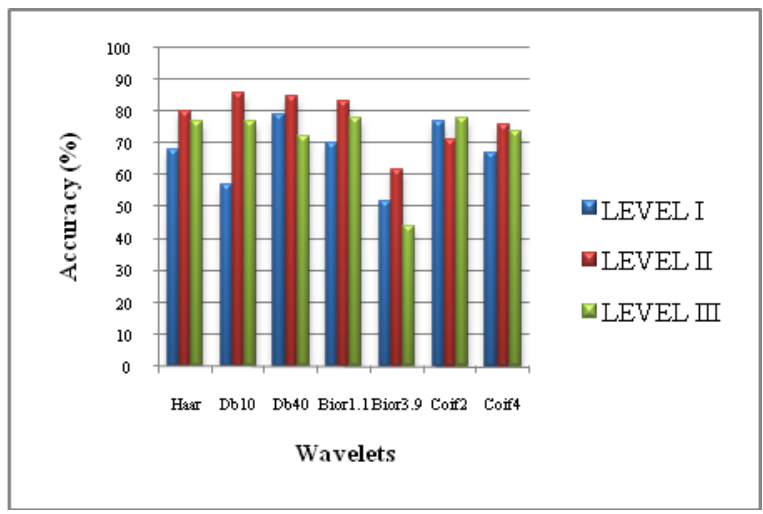

Fig. 7. Comparison of Discrete Wavelet Transform Levels

\section{CONCLUSION}

The objective of this project was to devise an algorithm to research ear recognition techniques. The recognition rate was calculated by varying the coefficients in DCT and DFT, and by varying levels in DWT. The best accuracy that was obtained was 86\%, using 2nd level DWT (with db10 wavelet). Using DCT and DFT an accuracy rate of $83 \%$ and $81 \%$ was obtained, respectively. However, better results can be achieved if the database used is of better resolution and pictures taken are at an appropriate angle in a well-lit background. The processor used was - 8th Gen Intel Core i7 processor, 3.40GHz base processor speed.

\section{REFERENCES}

1. BURGE, M. AND BURGER, W. 1997. Ear biometrics for machine vision. In Proceedings of the 21st Workshop of the Austrian Association for Pattern Recognition.J. Clerk Maxwell, A Treatise on Electricity and Magnetism, 3rd ed., vol. 2. Oxford: Clarendon, 1892, pp.68-73.

2. Ansari, S. and Gupta, P., "Localization of ear using outer helix curve of the ear," in [the International Conference on Computing: Theory and Applications (ICCTA)], 688-692 (2007).

3. MORENO, B., SANCHEZ, A., AND VELEZ, J. 1999. On the use of outer ear images for personal identification in security applications. In Proceedings of the 33rd IEEE International Conference on Security Technology. 469-476.

4. Anam Tariq, M. Almas Anjumt, M. Usman Akram, 2011. Personal Identification Using Computerized Human Ear Recognition System. In Proceedings of 2011 International Conference on Computer Science and Network Technology

5. FENG, J. AND MU, Z. 2009. Texture analysis for ear recognition using local feature descriptor and transform filter. Proc. SPIE 7496, 1.

6. WANG, Y., MU, Z., AND ZENG, H. 2008. Block-Based and multi-resolution methods for ear recognition using wavelet transform and uniform local binary patterns. In Proceedings of the 19th IEEE International Conference on Pattern Recognition (ICPR). 1-4.

7. Karuna Soni, Umesh Kumar, Sandeep K. Gupta, Shubh. L. Agrwal, 2014. A New Gabor Wavelet Transform Feature Extraction Technique for Ear Biometric Recognition. In 2014 6th IEEE Power India International Conference (PIICON)

8. ABATE, A., NAPPI, M., RICCIO, D., AND RICCIARDI, S. 2006. Ear recognition by means of a rotation invariant descriptor. In Proceedings of the 18th IEEE International Conference on Pattern Recognition (ICPR). 437-440.

9. S. Taertulakarn, C. Pintavirooj, P. Tosranon, K. Hamamoto, 2016. The preliminary investigation of ear recognition using hybrid technique. In 2016 9th Biomedical Engineering International Conference (BMEiCON).

10. Bao-Qing Zhang, Zhi-Chun Mu, Hui Zeng, Hong-bo Huang, 2013. Ear recognition based on Gabor scale information. In 2013 International Conference on Wavelet Analysis and Pattern Recognition.

11. Shubhangi Khobragade ; Dheeraj Dilip Mor ; Aman Chhabra, 2015. A method of ear feature extraction for ear biometrics using MATLAB. In 2015 Annual IEEE India Conference (INDICON).
12. Li Yuan, Zhi-chun Mu, 2007. Ear Recognition based on 2D Images. In 2007 First IEEE International Conference on Biometrics: Theory, Applications, and Systems

13. Shrikant Tiwari, Santosh Kumar, Sanjay Kumar, G R Sinha, 2015. Ear recognition for newborns. In proceedings of 2015 2nd International Conference on Computing for Sustainable Global Development (INDIACom)

14. Ping Yan, Kevin W. Bowyer, 2007. Biometric Recognition Using 3D Ear Shape. In IEEE Transactions on Pattern Analysis and Machine Intelligence ( Volume: 29 , Issue: 8 , Aug. 2007)

15. T. Yuizono ; Y. Wang ; K. Satoh ; S. Nakayama, 2002. Study on individual recognition for ear images by using genetic local search. In proceedings of the 2002 Congress on Evolutionary Computation. CEC'02 (Cat. No.02TH8600)

16. Banafshe Arbab-Zavar ; Mark S. Nixon ; David J. Hurley, 2007. On Model-Based Analysis of Ear Biometrics. In 2007 First IEEE International Conference on Biometrics: Theory, Applications, and Systems

17. Esther Gonzalez ; Luis Alvarez ; Luis Mazorra, 2012. Normalization and feature extraction on ear images. In 2012 IEEE International Carnahan Conference on Security Technology (ICCST)

18. Muthukumar Arunachalam; Santham Bharathy Alagarsamy, 2017. An efficient ear recognition system using DWT \& BLPOC. In 2017 International Conference on Inventive Communication and Computational Technologies (ICICCT)

19. M. Oravec ; J. Pavlovicova, 2004. Face recognition methods based on principal component analysis and feedforward neural networks. In 2004 IEEE International Joint Conference on Neural Networks (IEEE Cat. No.04CH37541)

20. Asmaa Sabet Anwar ; Kareem Kamal A. Ghany ; Hesham ElMahdy, 2015. Human ear recognition using SIFT features. In 2015 Third World Conference on Complex Systems (WCCS)

21. MATLAB for Artificial Intelligence [Online]. Available: https://in.mathworks.com

22. Fast Fourier Transform (FFT) Algorithm [Online]. Available: https://allsignalprocessing.com/fast-fourier-transform-fft-algorithm

23. The Fourier Transform: Linking Time and Frequency Domains [Online]. Available: https://allsignalprocessing.com/the-fourier-transform

24. S. Salivahanan, "Digital Signal Processing", by McGraw Hill, 2015

25. S. Jayaraman, S. Esakkirajan, T Veerakumar, "Digital Image Processing", by McGraw Hill, 2008

26. Fig. https://users.cs.cf.ac.uk/Dave.Marshall/Multimedia/node231.html

27. Fig. 3. by Bob K - Own work, CC0, https://commons.wikimedia.org/w/index.php?curid=75689115

28. Fig.

https://dsp.stackexchange.com/questions/38183/edge-map-based-on-a -haar-wavelet-transform

\section{AUTHORS PROFILE}

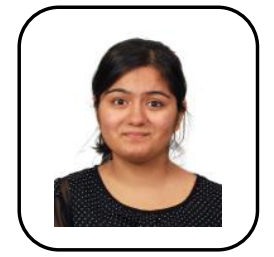

Ms. Aishna Sharma was born in Mumbai, Maharashtra in India on January $6^{\text {th }}$, 1998. She is currently pursuing her Bachelor of Engineering in the stream of Electronics and Telecommunication from Thadomal Shahani Engineering College (TSEC), Mumbai University. Her research interests include image processing, soft computing, database management systems, cryptography.

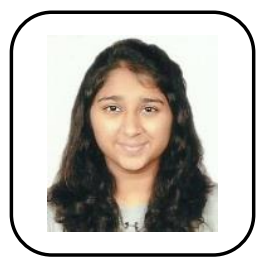

Ms. Natasha Lalwani was born in New Delhi in India on June $9^{\text {th }}, 1998$. She is currently pursuing her Bachelor of Engineering in the stream of Electronics and Telecommunication from Thadomal Shahani Engineering College (TSEC), Mumbai University. Her research interests include image processing, data science, database management systems, digital signal processing. 


\section{Biometric Identification using Human Ear}

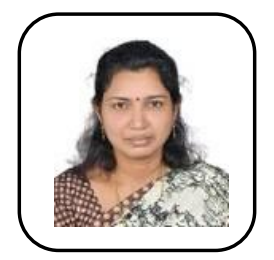

Dr. Mani Roja M. Edinburgh was born in Tirunelveli (T.N.) in India on June $19^{\text {th }}$, 1969. She has received BE in Electronics and Communication Engineering from GCE Tirunelveli, Madurai Kamraj University in 1990, and ME in Electronics from Mumbai University in 2002. She completed her PhD degree in 2015 from SGBA University. Her employment experience includes 29 years as an educationist at Thadomal Shahani Engineering College (TSEC), Mumbai University. She holds the post of a professor in TSEC. Her special fields of interest include Image Processing and Data Encryption. She has over 40 papers in National/International Conferences and Journals to her credit. She is a member of IETE and ISTE.

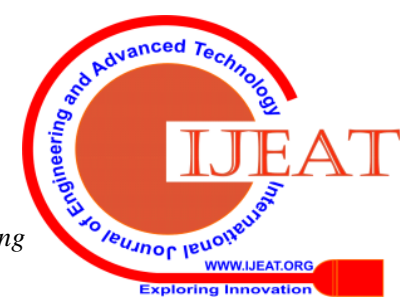

